\title{
Comparison of high-throughput techniques for metals purification and subsequent isotope analyses in modern applications
}

\author{
BRANDON MAHAN ${ }^{1}$ AND ANTHONY DOSSETO $^{2}$ \\ ${ }^{1}$ James Cook University \\ ${ }^{2}$ University of Wollongong \\ Presenting Author: brandon.mahan@jcu.edu.au
}

Metals—-such as $\mathrm{Ca}, \mathrm{Sr}, \mathrm{Fe}, \mathrm{Cu}$ and $\mathrm{Zn}$ - and their isotopes have become increasingly important (and popular) within many fields of research, and their application has broadened into "nonconventional" applications, such as in biomedicine, archaeology, anthropology, and ore exploration. Regardless of application, the common rate-limiting step for analyses in such isotopic systems is the isolation of the element of interest for high resolution isotopic characterization (generally by multi-collector inductively coupled plasma mass spectrometry, or MC-ICP-MS), which is conventionally done using time-consuming gravimetric ion exchange chromatography. The propagation of this convention hinders productivity, in that the number of unique samples that can feasibly be measured by an operator, or within a given project, is largely beholden to the duration of the complete element purification protocol for a typical sample batch (usually less than 20 samples at a time). This in turn relegates much of the academic personnel's time to sample preparation. Cumulatively, this (i) results in limited statistics due to small datasets, (ii) exacerbates the limitations imposed on scientific efficacy via time/energy consumption, (iii) can hinder the viability of isotope geochemistry for certain applications, all while (iv) often rendering isotope analyses prohibitively expensive.

In recent years, this acute problem has sparked growing interest in the development of element purification protocols that alleviate such issues by significantly decreasing the ion exchange purification process through increasing reagent flow rates (e.g. through vacuum, pressurization or centrifugation), and/or by automating this process in part or in full. Here, we discuss both existing and novel protocols for high throughput element purification (with a focus on transition metals), and compare their throughput power, advantages and disadvantages, and the application context(s) wherein they may be most synergistic. 